\title{
DISPLACEMENT OF THE ORBITAL FLOOR AND TRAUMATIC DIPLOPIA*
}

\author{
BY
}

\section{T. KEITH LYLE \\ London}

Although diplopia resulting from head injury is more usually due to a lesion of one of the ocular motor nerves, it may be caused by direct injury to the orbit. Sometimes damage occurs to one or more of the extrinsic ocular muscles, especially the obliques, because of their attachments to the bone in the anterior part of the orbit, but more frequently the injury brings about an alteration in the position of the eye, which alters the direction of its visual axis, causing a positional heterotropia or strabismus. If the orbital floor is displaced downwards, as so commonly occurs in fracture of the malar and maxilla, the eyeball is also displaced downwards and its visual axis is usually directed downwards; this leads to hypotropia of the affected eye and a relative defect of its power of elevation (Fig. 1).

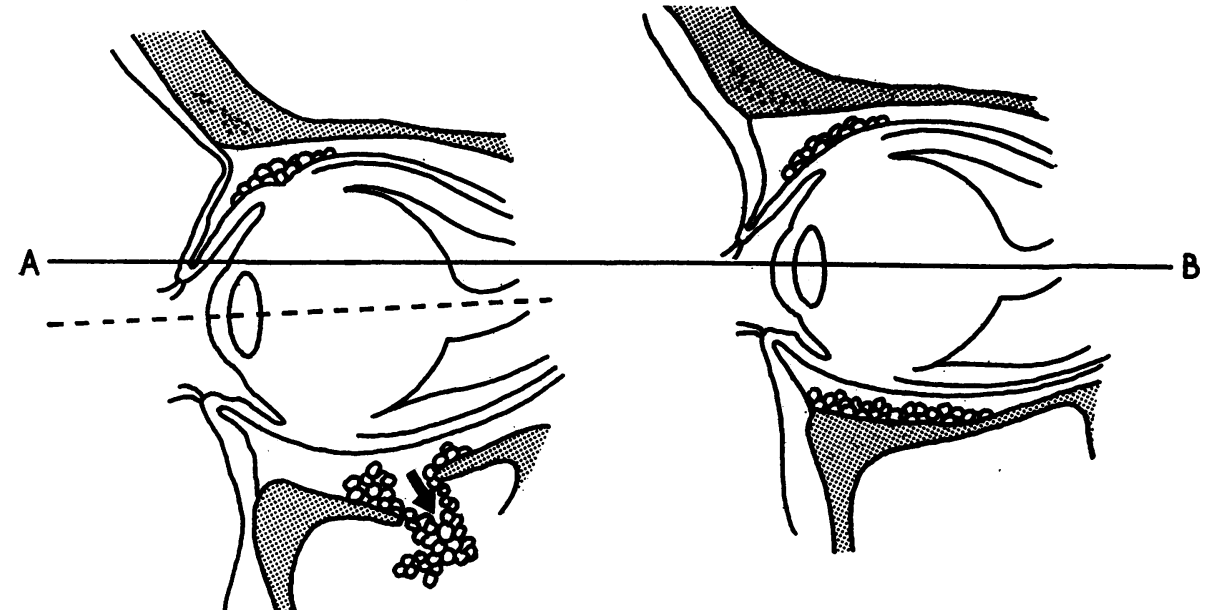

FIG. 1.-Displacement of eyeball and visual axis due to fracture of orbital floor (left); normal position (right). The line AB represents the visual axis in the normal position and the dotted line that in the displaced position.

\section{Clinical Material}

This communication is based on eighteen cases, sixteen of which were briefly mentioned in a recent article (Lyle, 1959).

All these patients had constant vertical diplopia, except one in whom vertical diplopia occurred only when looking upwards In some cases there was an obvious displacement of the eyeball as a whole, but in others the displacement was so slight as not to be apparent.

\footnotetext{
* Received for publication April 4, 1960.
} 


\section{Effect on the Eyeball of Displacement of the Orbital Floor}

It is not always realized that, although there may be a gross displacement of the eyeball as a whole, there may be no displacement of the visual axis and hence no diplopia. This is illustrated in Figs 2 and 3 (Lyle, 1949).

Fig. 2 shows a patient with gross displacement of the orbital floor and eyeball on the right side caused by an orbital injury; in spite of this displacement the visual axes were parallel not only in the primary position (as shown by the Maddox rod test), but over the entire visual field. The eyeball was certainly displaced but the visual axes had remained parallel.

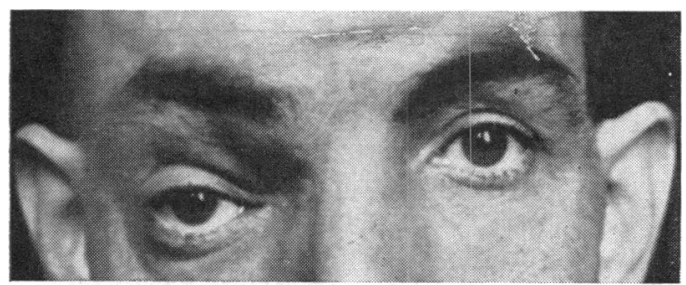

FIG. 2.-Example of gross displacement of orbital floor and right eyeball, with no accompanying diplopia (from Lyle, 1949).

By contrast, Fig. 3 shows a patient with a slight displacement of the eyeball caused by a fracture of the right maxilla and malar bones, who had constant vertical diplopia and $6 \Delta$ of right hypotropia as measured by the Maddox rod test. The diplopia was relieved by operation. He had a relative paresis of the right superior rectus with greatest vertical separation of the images on dextro-elevation. The diplopia disappeared after the visual axes had been rendered parallel by means of a myectomy of the left inferior oblique muscle.

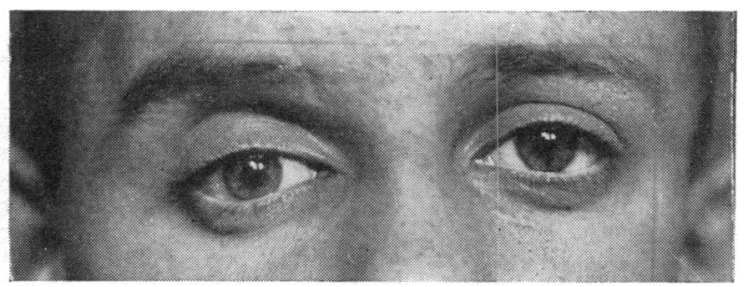

FIG. 3.-Example of slight displacement of right eyeball, with constant vertical diplopia (from Lyle, 1949).

\section{Orbital Reconstruction}

In considering the treatment of these cases of traumatic diplopia, it should be remembered that the patients had invariably been referred on account of the diplopia. In most cases expert surgical treatment had already been undertaken for the attempted correction of the bony displacement (or had been deemed contraindicated). Attention was therefore directed to the alleviation of the double vision by means of surgical adjustment of the extrinsic ocular muscles rather than to the improvement of the cosmetic appearance. It should perhaps be mentioned that, at the time of the accident, which had usually been a serious one and accompanied by severe concussion and other injuries such as fractures of long bones and damage to the chest. the chief concern had been to keep the patient alive, so that reduction of fractures of malar and maxilla involving the 
orbital floor (if such were feasible) had not always been attempted at the time when it might have been possible. Furthermore, the detection of displacement of the bones around the orbit may be extremely difficult when there is much diffuse swelling and bruising of the tissues of the face.

Duke-Elder (1954) states:

The healing of the orbital and facial bones is so rapid that if repositioning, which can usually be accomplished by closed methods of manipulation soon after the accident, is not done at an early stage and the fragments are allowed to consolidate in their abnormal position, considerable force must be used to refracture and replace them or, alternatively, major surgical procedures sometimes on a heroic scale are necessary to get rid of the deformity. Whenever it is possible, therefore, repositioning should be undertaken as soon as the period of shock is passed and the general condition of the patient allows, even before the local swelling of the lids and face has subsided.

McIndoe (1941) considered that manipulation, disimpaction, and the use of plaster caps, splints, etc., is possible before the end of the third month. If the orbital floor is depressed by fracture of the orbital plate of the maxilla without displacement of the malar and maxilla as a whole, access may sometimes be obtained through the antrum by the Caldwell-Luc approach, and the loose fragments of bone and blood clot may be removed and the floor elevated (Lyle, 1941). It may be necessary to retain the fractured bone in place by temporarily packing the antrum with gauze or by the insertion of an inflated rubber balloon.

Late treatment by camouflage operations may be required to disguise the considerable deformity and to raise the floor of the orbit if the repositioning of the displaced fragments is delayed beyond 3 months. Defects can be overcome by the use of grafts of bone or cartilage (Mowlem, 1941), or by the use of plastic resins or glass wool.

Although there is much to be said for raising the effective floor of the orbit by implanting subperiosteally a piece of cartilage, decalcified bone, or strips of fascia lata, it is not always found that, where one of these manoeuvres has been carried out, the diplopia has completely disappeared; in fact, in one case, the diplopia was made worse by causing a limitation of downward movement, although admittedly the patient's appearance had been improved. In this series of cases no operation to raise the orbital floor was undertaken, the primary concern was to overcome the diplopia, and in all cases freedom from diplopia over the greater part of the binocular field was achieved.

\section{Surgery of the Extrinsic Ocular Muscles}

The correction of vertical strabismus caused by orbital trauma by means of operation upon the extrinsic ocular muscles was described by Lyle (1941) and a series of cases treated showing satisfactory results was reported. This subject was also dealt with by Neely $(1945,1947)$, Cross (1945), and Lyle and Cross (1951), who referred to the treatment of specific ocular palsies.

\section{Analysis of Present Series of Cases}

The eighteen cases reported here have been divided into four groups according to the nature of the fracture:

(1) Unilateral Fracture of Malar and Maxilla-the Affected Eye being Hypotropic (Cases 1-8: Table I, overleaf).

The displacement of the eyeball downwards tends to place the elevating muscles, especially the superior rectus, at a mechanical disadvantage, hence the "apparent" or "relative" defect of elevation. Since there is no actual ocular palsy, there is relatively little (if any) contracture of the direct antagonist and no gross overaction of the 
contralateral synergist. Thus, in most cases, the operation of choice is one designed to strengthen the affected muscle, i.e. by resection of the superior rectus. It may, however, be necessary in addition (or sometimes alternatively) to weaken its direct antagonist, the ipsilateral inferior rectus, by means of a small recession, and in some cases to weaken the

TABLE I

UNILATERAL FRACTURE OF MALAR AND MAXILLA, AFFECTED

\begin{tabular}{|c|c|c|c|c|c|}
\hline $\begin{array}{l}\text { Case } \\
\text { No. }\end{array}$ & Sex & $\begin{array}{c}\text { Age } \\
\text { (yrs) }\end{array}$ & Cause and Type of Injury & Extent and Type of Diplopia & $\begin{array}{c}\text { Side } \\
\text { Affected }\end{array}$ \\
\hline 1 & $\mathbf{M}$ & 42 & Fall from scaffolding & $\begin{array}{l}\text { Vertical ahead and upwards } \\
\text { Also obstruction of right } \\
\text { naso-lacrimal duct with } \\
\text { constant watering of right } \\
\text { eye }\end{array}$ & Right \\
\hline 2 & $\mathbf{M}$ & 30 & Cricket ball injury & Constant vertical & Right \\
\hline 3 & $\mathbf{M}$ & 48 & Motor cycle accident (driver) & $\begin{array}{l}\text { Constant vertical } \\
\text { Also gross loss of visual field } \\
\text { caused by bilateral optic } \\
\text { nerve lesion }\end{array}$ & Right \\
\hline 4 & $\mathbf{M}$ & 20 & $\begin{array}{l}\text { Motor scooter accident } \\
\text { (driver) }\end{array}$ & $\begin{array}{l}\text { Constant } \\
\text { Vertical (Fig. 5) }\end{array}$ & Left \\
\hline 5 & $\mathbf{M}$ & 32 & Motor accident (passenger) & $\begin{array}{l}\text { Constant } \\
\text { Vertical }\end{array}$ & Left \\
\hline 6 & $\mathbf{M}$ & 30 & Knocked down by truck & $\begin{array}{l}\text { Constant } \\
\text { Vertical }\end{array}$ & Left \\
\hline 7 & F & 36 & Motor accident (passenger) & $\begin{array}{l}\text { Constant } \\
\text { Vertical and horizontal }\end{array}$ & Left \\
\hline 8 & $\mathbf{F}$ & 44 & Motor accident (passenger) & $\begin{array}{l}\text { Constant } \\
\text { Vertical and horizontal }\end{array}$ & Left \\
\hline
\end{tabular}


elevation of the unaffected eye by a myectomy or recession of the contralateral inferior oblique or by a recession of the contralateral superior rectus. It is important to aim at securing ocular movements which are symmetrical and equal in all directions. Details relating to these eight cases are given in Table I. Figs 4-7 (overleaf) relate to Cases 2 and 4.

EYE HYPOTROPIC, BUT WITH VISUAL ACUITY 6/6 AND N5

\begin{tabular}{|c|c|c|c|c|}
\hline \multicolumn{2}{|c|}{ Pre-operative } & \multirow{2}{*}{$\begin{array}{l}\text { Interval } \\
\text { after } \\
\text { Injury }\end{array}$} & \multirow[b]{2}{*}{ Surgical Treatment } & \multirow{2}{*}{$\begin{array}{l}\text { Residual Deviation* } \\
\text { (if any) }\end{array}$} \\
\hline $\begin{array}{l}\text { Defective } \\
\text { Ocular } \\
\text { Movement }\end{array}$ & $\begin{array}{l}\text { Angle of Deviation* } \\
\text { in Primary Position }\end{array}$ & & & \\
\hline Elevation & Hypotropia $7 \triangle$ & $11 \mathrm{mths}$ & $\begin{array}{l}\text { Resection right superior } \\
\text { rectus } \\
\text { Right dacrocystorhino- } \\
\text { stomy }\end{array}$ & $\begin{array}{l}\text { Binocular single vision } \\
\text { Right hypophoria } 2 \Delta \\
\text { No watering }\end{array}$ \\
\hline Elevation & $\begin{array}{l}\text { Hypotropia } 6 \Delta \\
\text { Esotropia 4 } \triangle \\
\text { (Fig. 4A) }\end{array}$ & $15 \mathrm{mths}$ & $\begin{array}{l}\text { Resection right superior } \\
\text { rectus }\end{array}$ & $\begin{array}{l}\text { Binocular single vision } \\
\text { No vertical deviation } \\
\text { Right esophoria } 2 \triangle \\
\text { (Fig. 4B) }\end{array}$ \\
\hline Elevation & $\begin{array}{l}\text { Hypotropia } 9 \Delta \\
\text { Esotropia } 3 \Delta\end{array}$ & $18 \mathrm{yrs}$ & $\begin{array}{l}\text { Resection right superior } \\
\text { rectus }\end{array}$ & $\begin{array}{l}\text { No horizontal deviation } \\
\text { Right hyperphoria } 1 \Delta \\
\text { Peripheral fusion }\end{array}$ \\
\hline Elevation & $\begin{array}{l}\text { Hypotropia } 23 \Delta \\
\text { Exotropia } 6^{\circ} \\
\text { Excyclotropia } 3^{\circ} \\
\text { (Fig. 7A) }\end{array}$ & $8 \mathrm{mths}$ & $\begin{array}{l}\text { Resection left superior } \\
\text { rectus } 6 \mathrm{~mm} \text {. } \\
\text { (Fig. } 7 \mathrm{~B} \text { ) } \\
\text { Recession right superior } \\
\text { rectus } 3 \mathrm{~mm} \text {. } \\
\text { (Fig. 7C) }\end{array}$ & $\begin{array}{l}\text { Binocular single vision } \\
\text { Orthophoria } \\
\text { (Fig. 6) }\end{array}$ \\
\hline Elevation & $\begin{array}{l}\text { Hypotropia } 13 \Delta \\
\text { Esotropia } 6 \Delta\end{array}$ & 2 yrs & $\begin{array}{l}\text { Resection left superior } \\
\text { rectus }\end{array}$ & $\begin{array}{l}\text { Binocular single vision } \\
\text { Orthophoria }\end{array}$ \\
\hline Elevation & Hypotropia $21 \triangle$ & $9 \mathrm{mths}$ & $\begin{array}{l}\text { Resection left superior } \\
\text { rectus } \\
\text { Myectomy right inferior } \\
\text { oblique } \\
\text { Resection right inferior } \\
\text { rectus }\end{array}$ & $\begin{array}{l}\text { Binocular single vision } \\
\text { Left hypophoria } 3 \Delta\end{array}$ \\
\hline Elevation & $\begin{array}{l}\text { Hypotropia } 17 \triangle \\
\text { Esotropia } 9 \triangle\end{array}$ & $18 \mathrm{mths}$ & $\begin{array}{l}\text { Recession left inferior } \\
\text { rectus } \\
\text { Recession left medial rectus } \\
\text { Recession right inferior } \\
\text { rectus }\end{array}$ & $\begin{array}{l}\text { Binocular single vision } \\
\text { Left hyphoria } 1 \Delta \\
\text { Left esophoria } 3 \Delta\end{array}$ \\
\hline Elevation & $\begin{array}{l}\text { Hypotropia } 12 \Delta \\
\text { Exotropia } 7 \Delta \\
\text { Excyclotropia } 3 \Delta\end{array}$ & 12 mths & $\begin{array}{l}\text { Resection left superior } \\
\text { rectus } \\
\text { Recession right lateral } \\
\text { rectus } \\
\text { Marginal myotomy right } \\
\text { medial rectus }\end{array}$ & $\begin{array}{l}\text { Binocular single vision } \\
\text { Orthophoria }\end{array}$ \\
\hline
\end{tabular}

* Maddox rod test at $6 \mathrm{~m}$. 


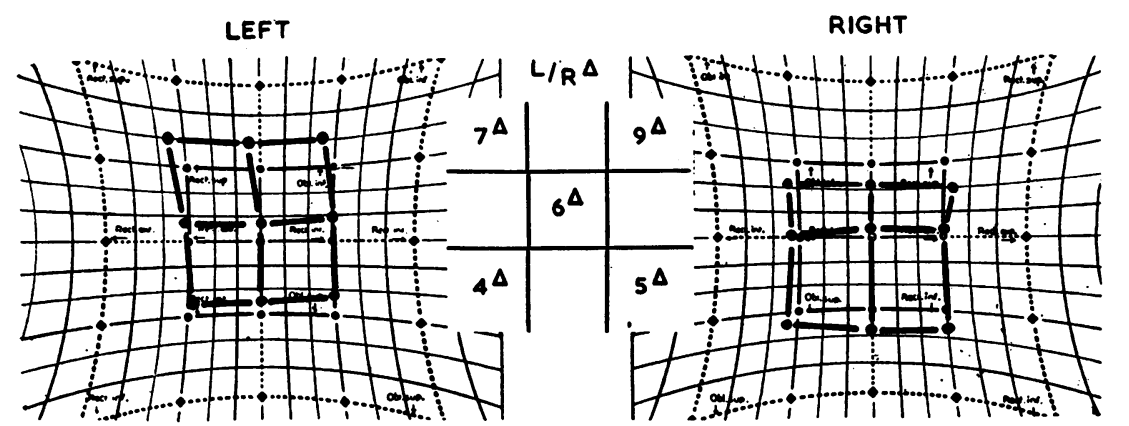

A

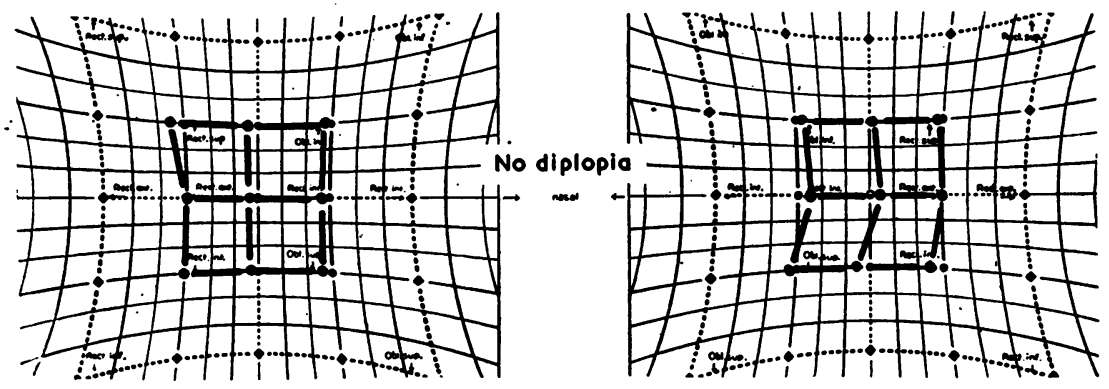

B

FIG. 4.-Case 2. Hess charts. (A) before operation, (B) after resection right superior rectus.(Table I).

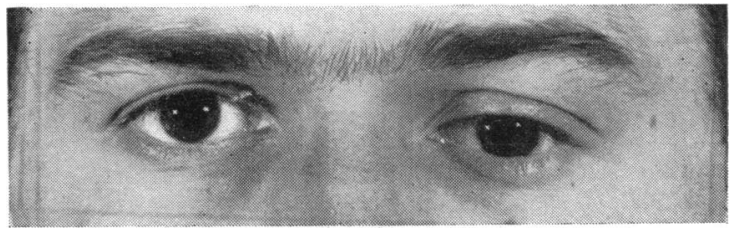

Fig. 5.-Case 4. Displacement of left eyeball with constant vertical diplopia.

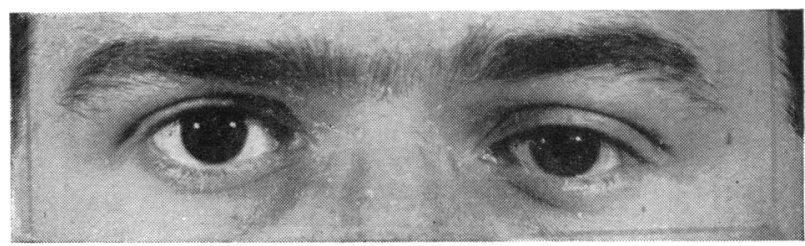

Fig. 6.-Case 4. Post-operative orthophoria with binocular single vision (Table I). 


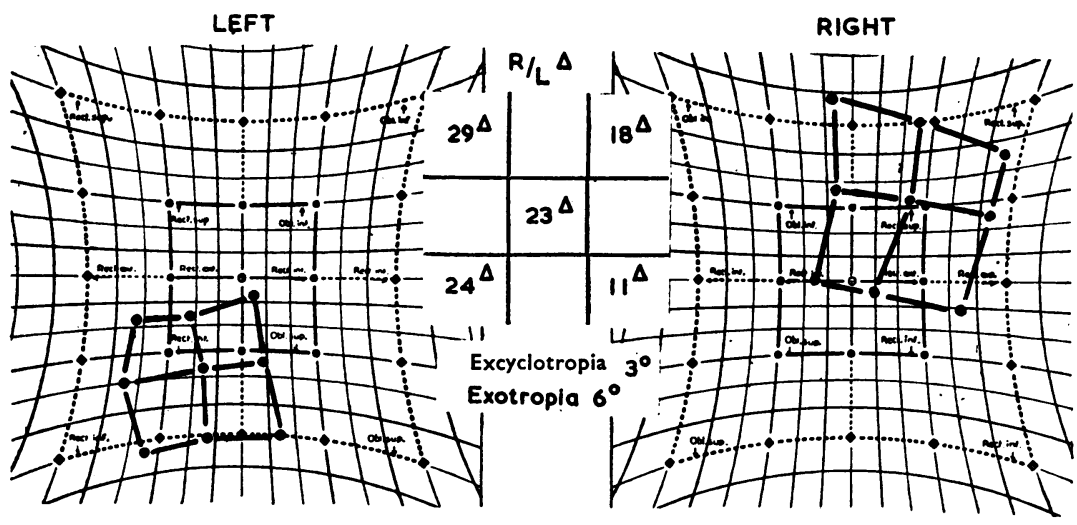

A

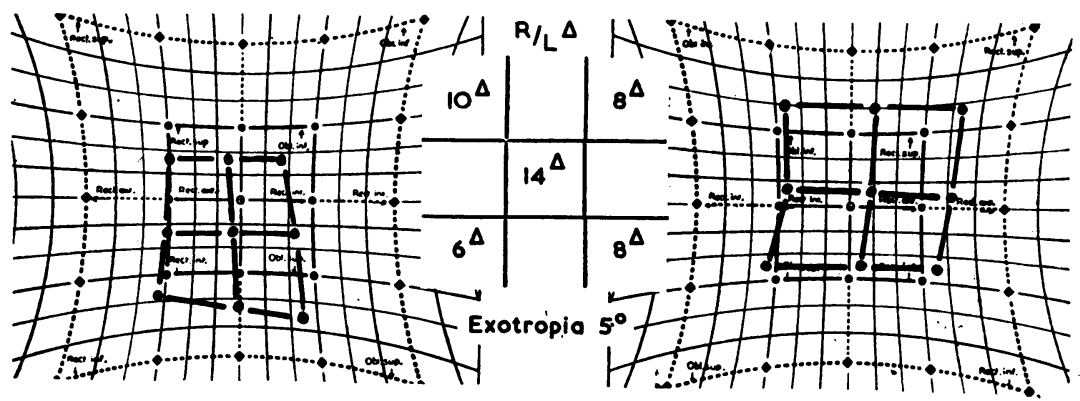

B

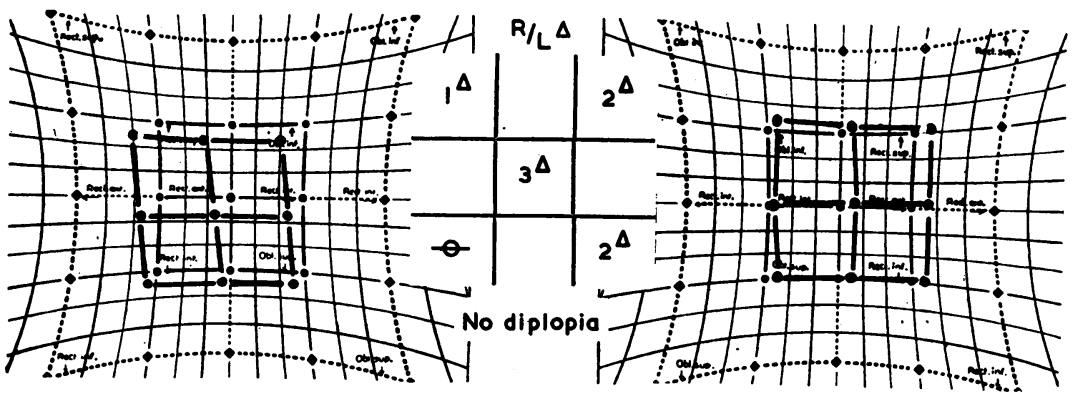

C

Fig. 7.-Case 4. Hess charts. (A) before operation, (B) after resection left superior rectus $6 \mathrm{~mm}$., (C) after recession right superior rectus $3 \mathrm{~mm}$. (Table I). 
(2) Unilateral Fracture of Malar, Maxilla, and Frontal Bone-the Affected Eye being Hypotropic (Cases 9-12: Table II).

In these cases there may be defective depression and/or defective horizontal movement in addition to the defective elevation which is usually of considerable degree; hence more complicated surgical procedures may be needed.

Details relating to these four cases are given in Table II. Fig. 8 (opposite) relates to Case 9, and Figs 9 and 10 (overleaf) to Cases 10 and 12.

TABLE II

UNILATERAL FRACTURE OF MALAR, MAXILLA, AND FRONTAL BONE. $\stackrel{\omega}{\oplus}$

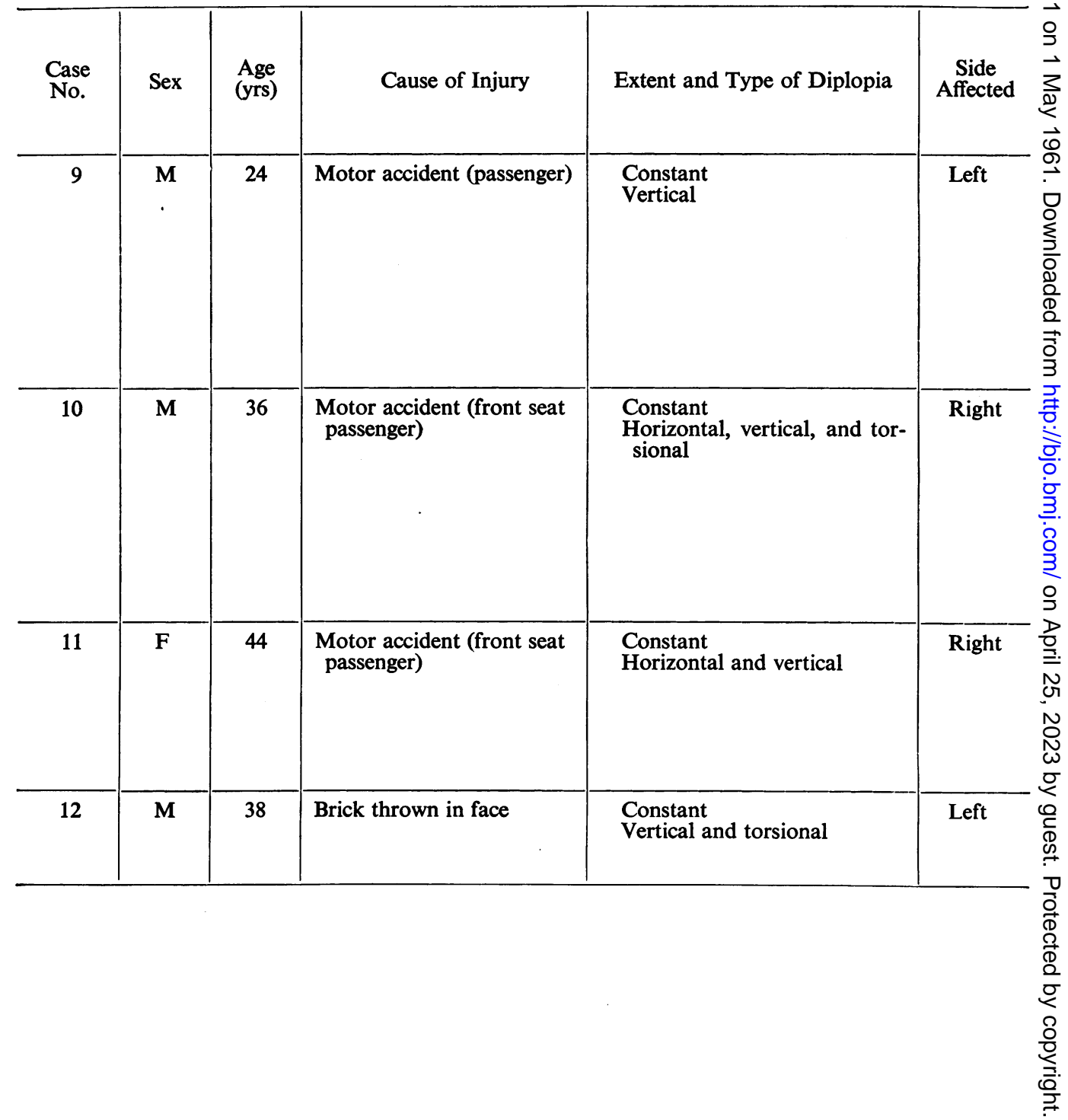



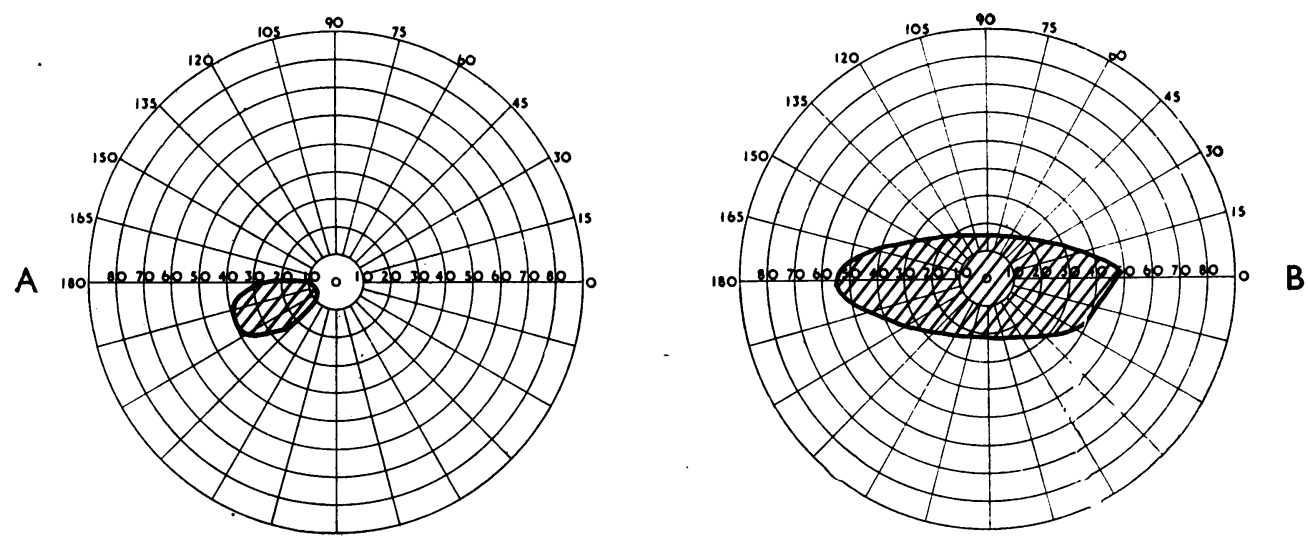

Fig. 8.-Case 9. Visual fields. (A) before operation, April 30, 1957, (B) after a series of operations (Table II), December 29, 1958.

\section{AFFECTED EYE HYPOTROPIC BUT WITH VISUAL ACUITY 6/6 AND N5}

\begin{tabular}{|c|c|c|c|c|}
\hline \multicolumn{2}{|c|}{ Pre-operative } & \multirow{2}{*}{$\begin{array}{l}\text { Interval } \\
\text { after } \\
\text { Injury }\end{array}$} & \multirow[b]{2}{*}{ Surgical Treatment } & \multirow{2}{*}{$\begin{array}{l}\text { Residual Deviation* } \\
\text { (if any) }\end{array}$} \\
\hline $\begin{array}{l}\text { Defective } \\
\text { Ocular } \\
\text { Movement }\end{array}$ & $\begin{array}{l}\text { Angle of Deviation* } \\
\text { in Primary Position }\end{array}$ & & & \\
\hline $\begin{array}{l}\text { Elevation } \\
\text { and de- } \\
\text { pression }\end{array}$ & $\begin{array}{l}\text { Hypotropia } 12 \Delta \\
\text { Exotropia } 4 \Delta \\
\text { Field of binocular } \\
\text { fixation restricted } \\
\text { (Fig. 8A) }\end{array}$ & 6 yrs & $\begin{array}{l}\text { Recession right superior } \\
\text { rectus } 3 \mathrm{~mm} \text {. } \\
\text { Recession right inferior } \\
\text { rectus } 3 \mathrm{~mm} \text {. } \\
\text { Myectomy right inferior } \\
\text { oblique } \\
\text { Recession right lateral } \\
\text { rectus } 5 \mathrm{~mm} \text {. } \\
\text { Marginal myotomy right } \\
\text { inferior oblique }\end{array}$ & $\begin{array}{l}\text { Binocular single vision in } \\
\text { central visual field } \\
\text { (Fig. 8B) } \\
\text { Left hypophoria } 3 \Delta\end{array}$ \\
\hline Elevation & $\begin{array}{l}\text { Hypotropia } 30 \Delta \\
\text { (Fig. 9A) }\end{array}$ & 2 mths & 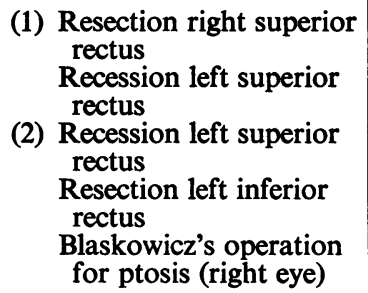 & $\begin{array}{l}\text { Binocular single vision - } \\
\text { Orthophoria } \\
\text { (Fig. 9B) }\end{array}$ \\
\hline $\begin{array}{l}\text { Elevation, } \\
\text { depres- } \\
\text { sion, and } \\
\text { abduction }\end{array}$ & $\begin{array}{l}\text { Hypotropia } 4 \Delta \\
\text { Exotropia } 45 \Delta\end{array}$ & 2 yrs & $\begin{array}{l}\text { Recession left superior } \\
\text { rectus } \\
\text { Recession left lateral rectus } \\
\text { Recession right lateral } \\
\text { rectus } \\
\text { Resection right medial } \\
\text { rectus }\end{array}$ & $\begin{array}{l}\text { Binocular single vision in } \\
\text { central visual field } \\
\text { Right hypophoria } 2 \Delta \\
\text { Esophoria } 4 \Delta\end{array}$ \\
\hline Elevation & $\begin{array}{l}\text { Hypotropia } 17 \Delta \\
\text { Excyclotropia } 5 \Delta \\
\text { (Fig. 10A) }\end{array}$ & 7 mths & $\begin{array}{l}\text { Resection left superior } \\
\text { rectus } 4 \mathrm{~mm} \text {. }\end{array}$ & $\begin{array}{l}\text { Binocular single vision } \\
\text { Orthophoria } \\
\text { (Fig. 10B) }\end{array}$ \\
\hline
\end{tabular}

* Maddox rod test at $6 \mathrm{~m}$. 

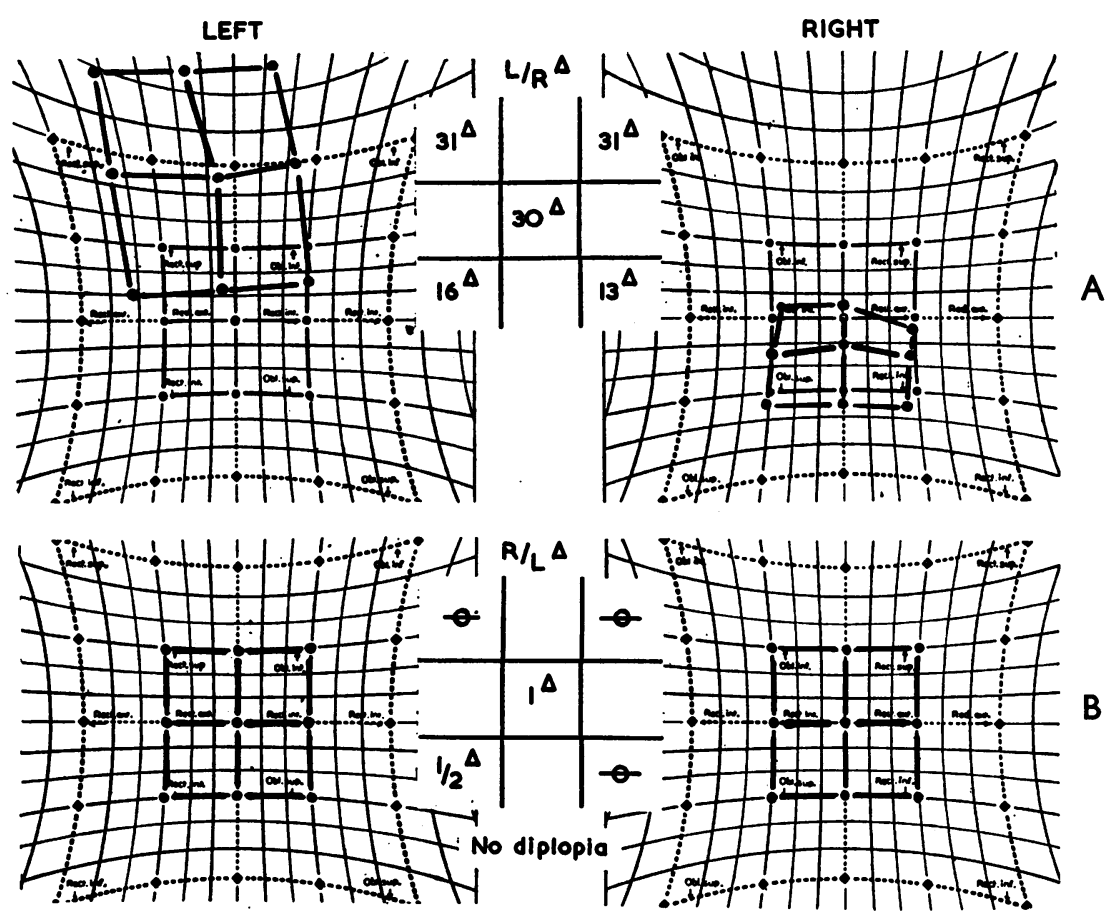

FIG. 9.-Case 10. Hess charts. (A) before operation, (B) after a series of operations (Table II).
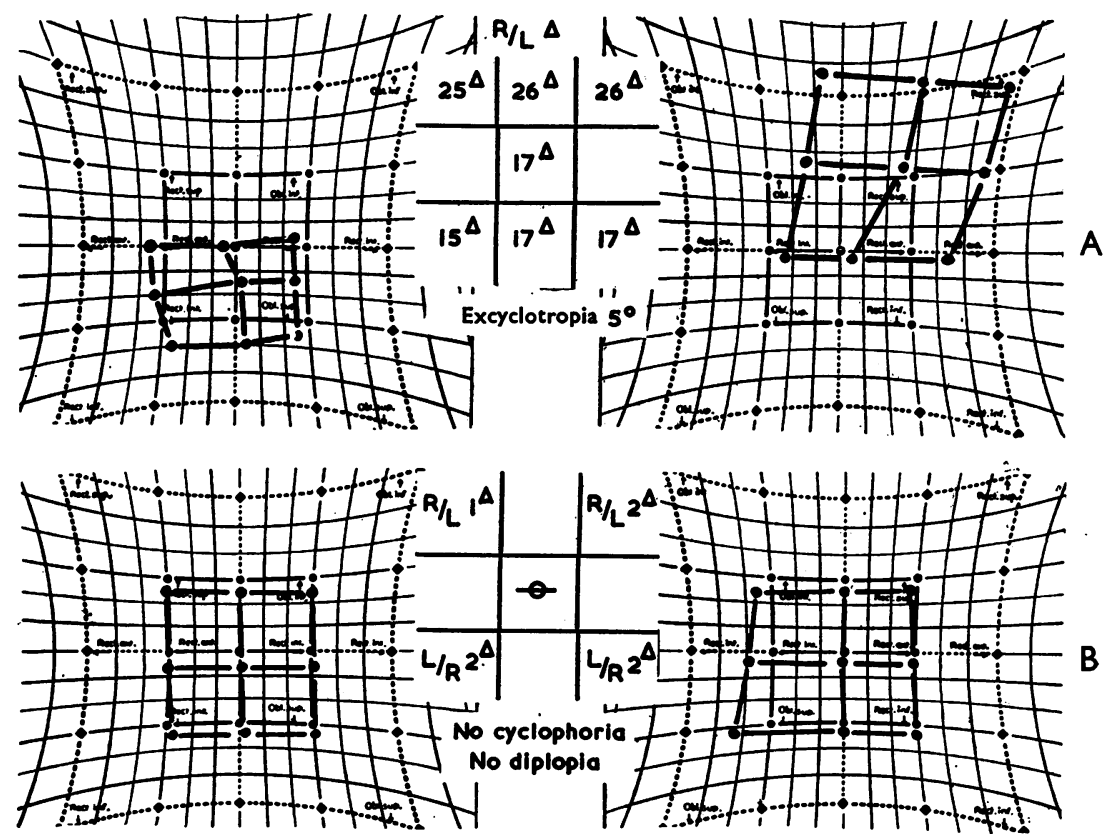

Fig. 10.-Case 12. Hess charts. (A) before operation, (B) after resection left superior rectus (Table II). 
(3) Unilateral Fracture of Malar and Maxilla with Defective Depression-the Affected Eye being Hypertropic (Cases 13 and 14: Table III).

The defective depression in these cases may be due to a thickening of the orbital floor, which causes a certain amount of mechanical displacement of the eyeball upwards with a resulting relative defect of depression.

Details relating to these two cases are given in Table III (overleaf). Fig. 11 relates to Case 14.
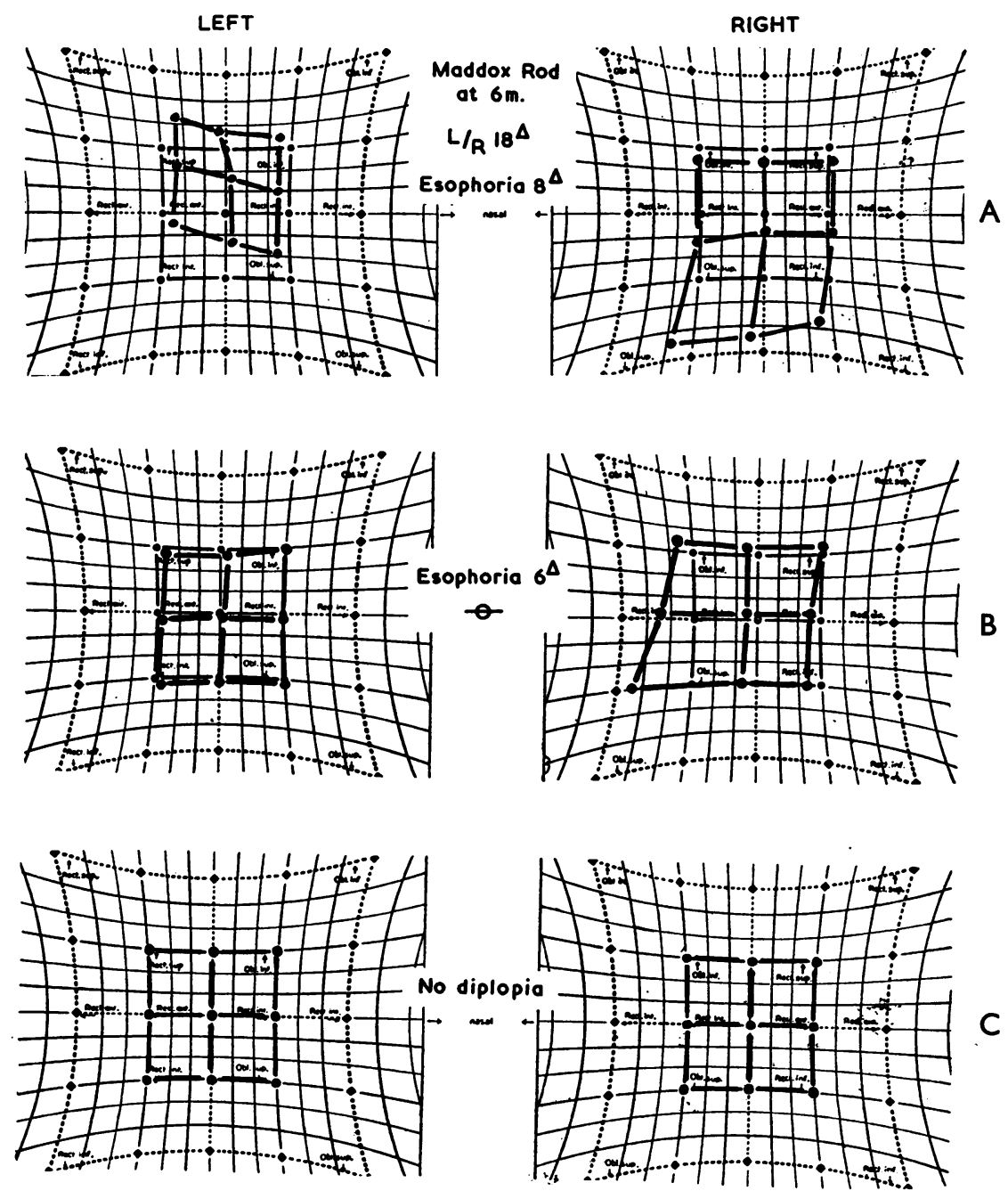

FIG. 11.-Case 14. Hess charts. (A) before operation, (B) after resection left inferior rectus, (C) after recession right medial rectus (Table III). 
TABLE III

UNILATERAL FRACTURE OF MAXILLA AND DEFECTIVE DEPRESSION

\begin{tabular}{c|c|c|l|l|c}
\hline $\begin{array}{l}\text { Case } \\
\text { No. }\end{array}$ & Sex & $\begin{array}{c}\text { Age } \\
\text { (yrs) }\end{array}$ & Cause of Injury & Extent and Type of Diplopia & $\begin{array}{c}\text { Side } \\
\text { Affected }\end{array}$ \\
\hline 13 & F & 56 & Fell on bucket & $\begin{array}{l}\text { Constant } \\
\text { Vertical }\end{array}$ & Right \\
\hline 14 & M & 39 & Motor accident & $\begin{array}{l}\text { Constant } \\
\text { Vertical }\end{array}$ & Left \\
\hline
\end{tabular}

(4) Central Naso-Maxillary Fracture leading to "Dish-face" Depression of the Root of the Nose (Cases 15-18: Table IV).

In those cases in which there is considerable displacement backwards of the fractured central naso-maxillary mass of bone there is, as one would expect, an increase of the interpupillary distance with resulting exotropia or exophoria. This latter deviation increases the torsional action of the oblique muscles and, since the inferior oblique is a stronger muscle than the superior oblique, there may be a certain amount of excyclotropia.

The disappearance of the excyclotropia in Case 16 (Fig. 13), as a result of overcoming the exotropia by a simple recession of the lateral rectus illustrates this point. On the other hand, when greater degrees of excyclotropia occur, more drastic procedures are needed, as shown in Case 17 (Fig. 14).

In all four cases of this group there was defective elevation of one eye as compared with the other. In three cases this discrepancy was sufficient to need surgical rectification.

Details relating to these four cases are shown in Table IV (overleaf). Fig. 12 (opposite) relates to Case 15, and Figs 13 and 14 (overleaf) to Cases 16 and 17. 
AFFECTED EYE HYPERTROPIC, BUT WITH VISUAL ACUITY 6/5 AND N5

\begin{tabular}{|c|c|c|c|c|}
\hline \multicolumn{2}{|c|}{ Pre-operative } & \multirow[b]{2}{*}{$\begin{array}{l}\text { Interval } \\
\text { after } \\
\text { Injury }\end{array}$} & \multirow[b]{2}{*}{ Surgical Treatment } & \multirow{2}{*}{$\begin{array}{l}\text { Residual Deviation* } \\
\text { (if any) }\end{array}$} \\
\hline $\begin{array}{l}\text { Defective } \\
\text { Ocular } \\
\text { Movement }\end{array}$ & $\begin{array}{l}\text { Angle of Deviation* } \\
\text { in Primary Position } \\
\text { (Maddox Rod } 6 \mathrm{~m} \text {.) }\end{array}$ & & & \\
\hline Depression & $\begin{array}{l}\text { Hypertropia } 29 \triangle \\
\text { Esotropia } 5 \Delta\end{array}$ & $4 \frac{1}{2}$ yrs & $\begin{array}{l}\text { Resection right inferior } \\
\text { rectus } \\
\text { Recession left inferior } \\
\text { rectus }\end{array}$ & $\begin{array}{l}\text { Binocular single vision } \\
\text { Right hyperphoria } 4 \Delta\end{array}$ \\
\hline \multirow[t]{2}{*}{ Depression } & \multirow[t]{2}{*}{$\begin{array}{l}\text { Hypertropia } 18 \Delta \\
\text { Esophoria } 8 \Delta \\
\text { (Fig. 11A) }\end{array}$} & \multirow[t]{2}{*}{$2 \mathrm{mths}$} & $\begin{array}{l}\text { Resection left inferior } \\
\text { rectus } \\
\text { (Fig. 11B) }\end{array}$ & \multirow[t]{2}{*}{ Binocular single vision } \\
\hline & & & $\begin{array}{l}\text { Recession right medial } \\
\text { rectus } \\
\text { (Fig. 11C) }\end{array}$ & \\
\hline
\end{tabular}

* Maddox rod test at $6 \mathrm{~m}$.
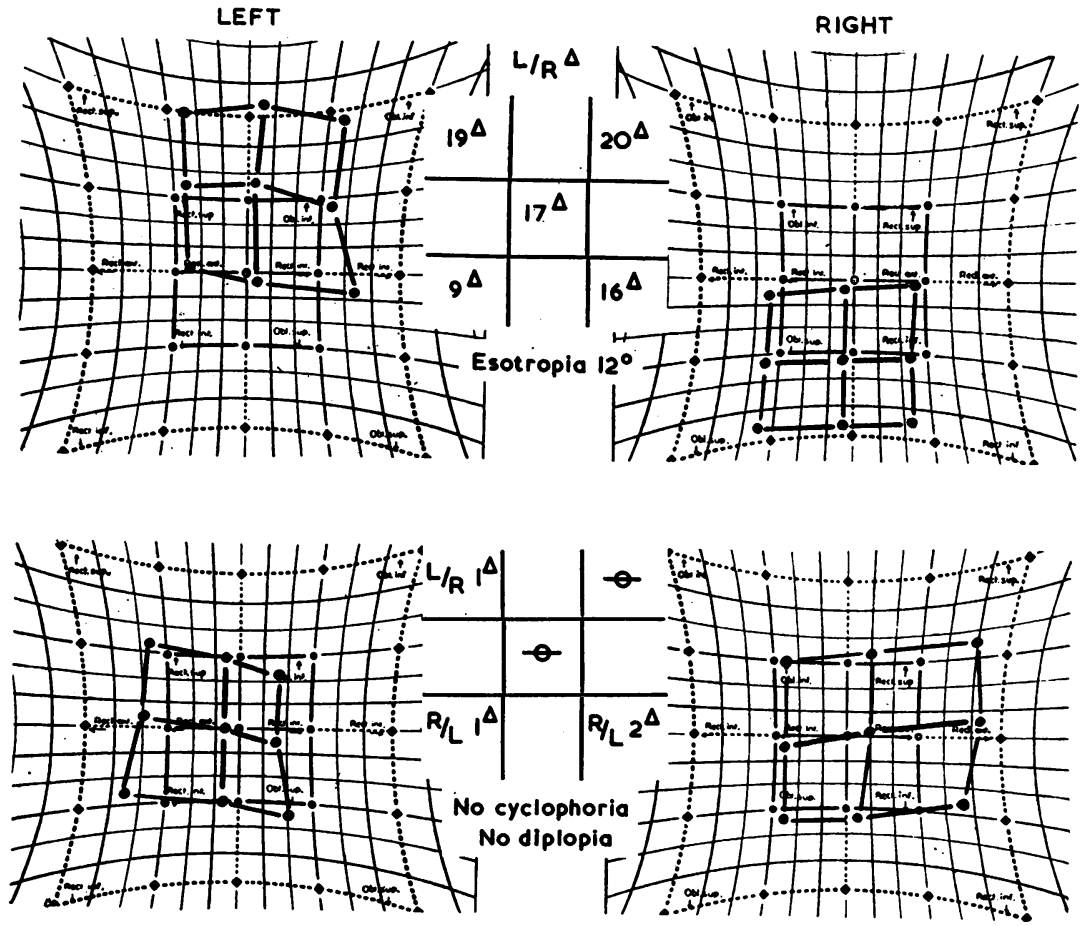

Fig. 12.-Case 15. Hess charts. (A) before operation, (B) after a series of operations 23

(Table (IV). 
TABLE IV

CENTRAL NASO-MAXILLARY FRACTURE.

\begin{tabular}{|c|c|c|c|c|c|}
\hline $\begin{array}{l}\text { Case } \\
\text { No. }\end{array}$ & Sex & $\begin{array}{l}\text { Age } \\
\text { (yrs) }\end{array}$ & Cause of Injury & Extent and Type of Diplopia & $\begin{array}{c}\text { Side } \\
\text { Affected }\end{array}$ \\
\hline 15 & $\mathbf{M}$ & 43 & Motor accident (passenger) & $\begin{array}{l}\text { Constant } \\
\text { Horizontal and vertical }\end{array}$ & Right \\
\hline 16 & $\mathbf{M}$ & 37 & Motor accident (driver) & $\begin{array}{l}\text { Constant } \\
\text { Horizontal, vertical and tor- } \\
\text { sional }\end{array}$ & $\begin{array}{l}\text { Right } \\
\text { Both }\end{array}$ \\
\hline 17 & $\mathbf{M}$ & 19 & Motor accident (passenger) & $\begin{array}{l}\text { Constant } \\
\text { Horizontal, vertical, and tor- } \\
\text { sional }\end{array}$ & $\begin{array}{l}\text { Right } \\
\text { Left }\end{array}$ \\
\hline 18 & $\mathbf{M}$ & 22 & Motor accident (passenger) & $\begin{array}{l}\text { Constant } \\
\text { Horizontal and vertical }\end{array}$ & Right \\
\hline
\end{tabular}

ब

舟

$\vec{\circ}$

$\vec{\omega}$

음.

cे

ơ

$\stackrel{\omega}{ \pm}$

옥

$\vec{z}$

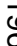

9

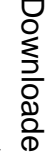

$\stackrel{\Phi}{\circ}$

웅

吾

응

음

음

으

음

今

$\omega$ 
VISUAL ACUITY 6/5 AND N/5 EACH EYE

\begin{tabular}{|c|c|c|c|c|}
\hline \multicolumn{2}{|c|}{ Pre-operative } & \multirow{2}{*}{$\begin{array}{l}\text { Interval } \\
\text { after } \\
\text { Injury } \\
\text { (mths) }\end{array}$} & \multirow{2}{*}{ Surgical Treatment } & \multirow{2}{*}{$\begin{array}{l}\text { Residual Deviation* } \\
\text { (if any) }\end{array}$} \\
\hline $\begin{array}{l}\text { Defective } \\
\text { Ocular } \\
\text { Movement }\end{array}$ & $\begin{array}{l}\text { Angle of Deviation* } \\
\text { in Primary Position }\end{array}$ & & & \\
\hline \multirow[t]{2}{*}{ Elevation } & \multirow[t]{2}{*}{$\begin{array}{l}\text { Hypotropia } 17 \Delta \\
\text { Esotropia } 12^{\circ} \\
\text { (Fig. 12A) }\end{array}$} & 8 & $\begin{array}{l}\text { Resection right superior } \\
\text { rectus } 4 \mathrm{~mm} \text {. } \\
\text { Recession right medial } \\
\text { rectus } 5 \mathrm{~mm} \text {. }\end{array}$ & . \\
\hline & & 10 & $\begin{array}{l}\text { Recession left medial rectus } \\
5 \mathrm{~mm} \text {. } \\
\text { Myectomy left inferior } \\
\text { oblique } \\
\text { Resection left lateral rectus } \\
5 \mathrm{~mm} \text {. } \\
\text { (Fig. 12B) }\end{array}$ & $\begin{array}{l}\text { Binocular single vision } \\
\text { Orthophoria }\end{array}$ \\
\hline $\begin{array}{l}\text { Elevation } \\
\text { Adduction }\end{array}$ & $\begin{array}{l}\text { Hypotropia } 3 \Delta \\
\text { Excyclotropia } 8^{\circ} \\
\text { Exotropia } 15^{\circ} \\
\text { (Fig. 13A) }\end{array}$ & 6 & $\begin{array}{l}\text { Recession left lateral rectus } \\
10 \mathrm{~mm} \text {. } \\
\text { (Fig. 13B) }\end{array}$ & $\begin{array}{l}\text { Binocular single vision } \\
\text { Right hypophoria } 3 \Delta \\
\text { No cyclophoria } \\
\text { No horizontal deviation }\end{array}$ \\
\hline Depression & \multirow{3}{*}{$\begin{array}{l}\text { Hypotropia } 17 \triangle \\
\text { Excyclotropia } 12^{\circ} \\
\text { Exotropia } 8^{\circ} \\
\text { (Fig. 14A) }\end{array}$} & 10 & $\begin{array}{l}\text { Resection right inferior } \\
\text { rectus } 4 \mathrm{~mm} \text {. } \\
\text { Recession right lateral } \\
\text { rectus } 6 \mathrm{~mm} \text {. } \\
\text { (Fig. 14B) }\end{array}$ & . \\
\hline \multirow[t]{2}{*}{ Elevation } & & 18 & $\begin{array}{l}\text { Bilateral myectomy inferior } \\
\text { obliques } \\
\text { (Fig. 14C) }\end{array}$ & \\
\hline & & & $\begin{array}{l}\text { Recession left lateral rectus } \\
\text { Recession left inferior } \\
\text { rectus } \\
\text { (Fig. 14D) }\end{array}$ & $\begin{array}{l}\text { Binocular single vision } \\
\text { Orthophoria }\end{array}$ \\
\hline $\begin{array}{l}\text { Elevation } \\
\text { and } \\
\text { Dextro- } \\
\text { depres- } \\
\text { sion }\end{array}$ & $\begin{array}{l}\text { Hypotropia } 17 \triangle \\
\text { Exotropia } 15^{\circ}\end{array}$ & 9 & $\begin{array}{l}\text { Resection right inferior } \\
\text { rectus } \\
\text { Recession right lateral } \\
\text { rectus } \\
\text { Blaskowicz' operation for } \\
\text { right ptosis }\end{array}$ & $\begin{array}{l}\text { Binocular single vision } \\
\text { Right hypophoria } 3 \Delta\end{array}$ \\
\hline
\end{tabular}

* Maddox rod test at $6 \mathrm{~m}$. 

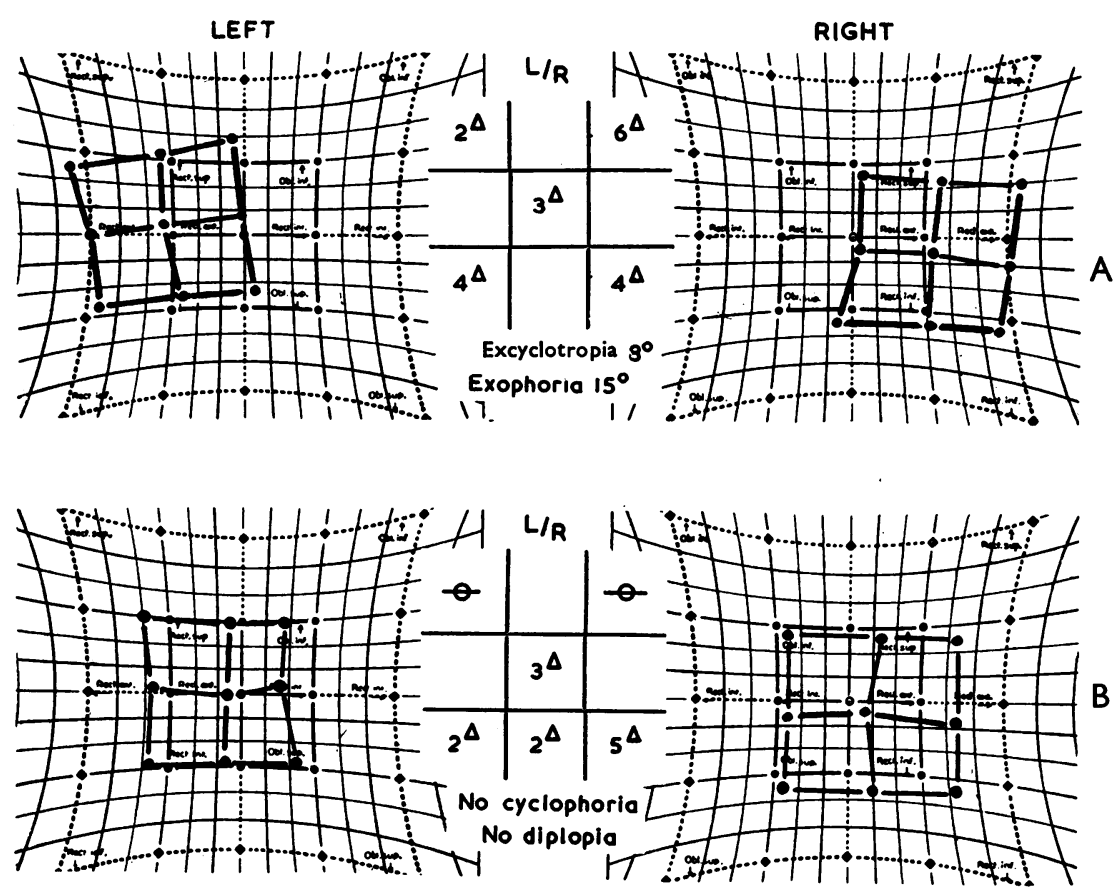

Fig. 13.-Case 16. Hess charts. (A) before operation, (B) after recession left lateral rectus $10 \mathrm{~mm}$. (Table IV).

\section{Summary}

A series of eighteen cases of fracture of the orbital floor with resulting diplopia is described. Restoration of normal ocular alignment and binocular single vision was achieved by surgery of the extrinsic ocular muscles.

\section{REFERENCES}

Cross, A. G. (1945). Trans. ophthal. Soc. U.K., 65, 20.

DUKE-ELDER, S. (1954). "Text-book of Ophthalmology", vol. 6. Kimpton, London

LYLE, T. KerTH (1941). Trans. ophthal. Soc. U.K., 61, 189.

(1949). Trans. ophthal. Soc. Austr., 9, 76.

(1959). Postgrad. Med., 25, 18.

and Cross, A. G. (1951). Brit. J. Ophthal., 35, 511.

MCINDOE, A. H. (1941). Brit. dent. J., 71, 235.

Mowlem, R. (1941). Brit. J. Surg., 29, 182.

NeELY, J. C. (1945). Trans. ophthal. Soc. U.K., 65, 47.

(1947). Brit. J. Ophthal., 31, 581. 
LEFT

RIGHT
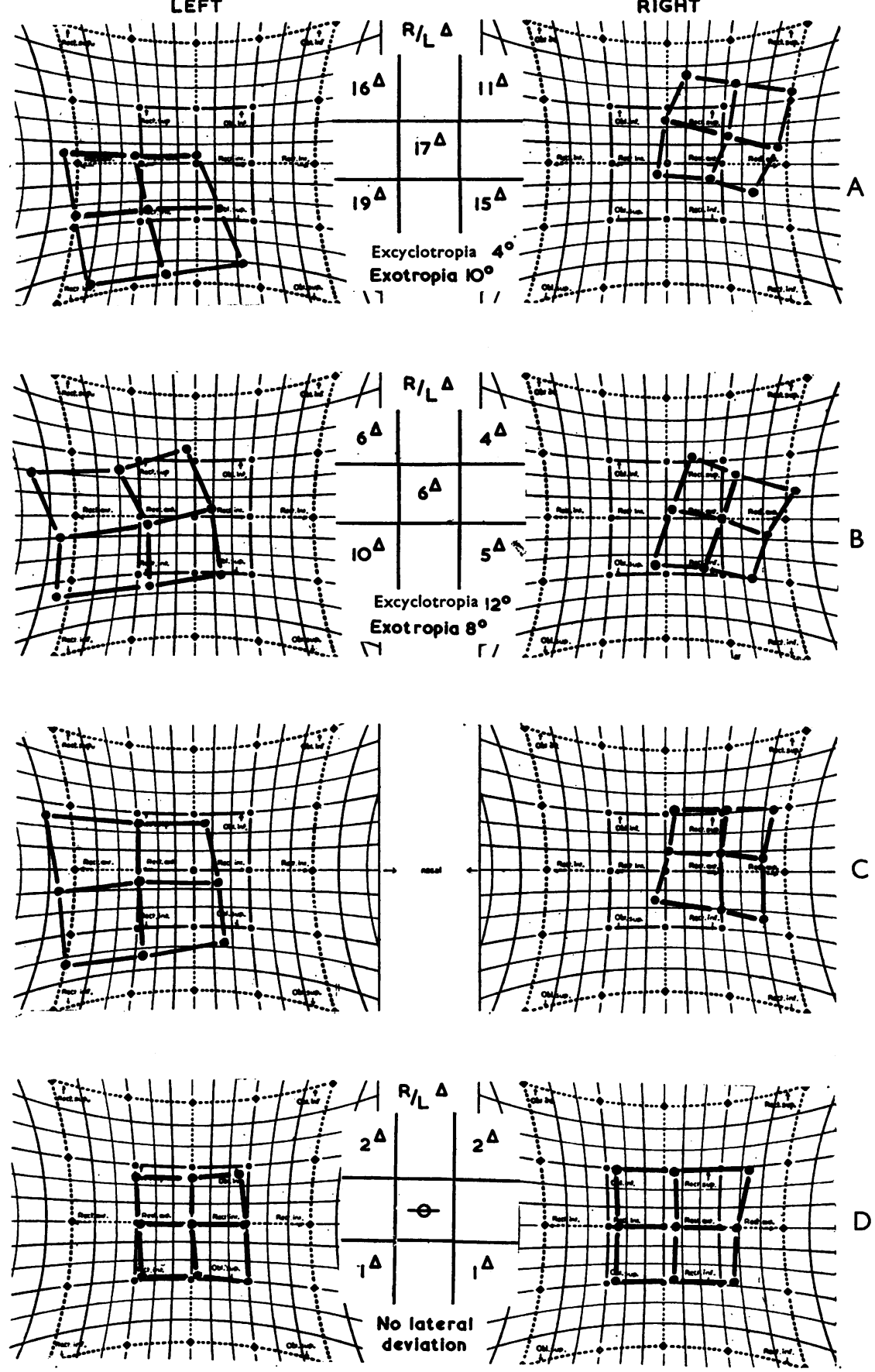

FIG. 14.-Case 17. Hess charts. (A) before operation, (B) after resection right inferior rectus $4 \mathrm{~mm}$. and recession right lateral rectus $6 \mathrm{~mm}$., (C) after bilateral myectomy inferior obliques, (D) after recession left lateral and inferior recti (Table IV). 